\title{
Análisis por medio de la normalización de variables para un modelo de planificación ambiental hídrica estacional
}

Analysis by means of variable normalization for a model of seasonal water environmental planning

Fecha de entrega: 26 de agosto 2015

Fecha de aceptación: 4 de noviembre 2016

\section{Juan Pablo Rodríguez ${ }^{1}$, Álvaro Suazo² e Iván Santelices ${ }^{3}$}

${ }^{1}$ Facultad del Medio Ambiente y Recursos Naturales, Universidad Distrital Francisco José de Caldas, Carrera 5 Este No 15 - 82, Avenida Circunvalar Venado de Oro, Bogotá D.C., Colombia, jprodriguezm@udistrital.edu.co

${ }^{2}$ Departamento de Ingeniería Civil y Ambiental, Universidad del Bío-Bío, Avenida Collao 1202, Casilla 5C, Concepción, Chile, asuazo@ubiobio.cl

${ }^{3}$ Escuela de Ingeniería Civil Industrial, Universidad del Bío-Bío, Avenida Collao 1202, Casilla 5C, Concepción, Chile, isanteli@ubiobio.cl

Se utiliza un procedimiento de normalización de variables y de construcción de un vector normalizado de calidad ambiental, lo cual permite estudiar la variación de la integralidad de los factores ambientales en la dinámica ambiental de una cuenca hídrica. Ello implica, la transformación de variables independientes que representan una situación compleja en una cuenca hidrográfica y su interacción con las plantas de tratamiento de aguas residuales. El método aplicado en este estudio es documental, explicativo y descriptivo mediante la recolección de información para la elaboración de la normalización de variables de calidad ambiental. Los resultados obtenidos establecen que los métodos de maximizar y minimizar aplicados al conjunto de datos, se ajustan a las variables de calidad ambiental analizados. Un análisis de los métodos de normalización presenta un resultado de aplicabilidad de un procedimiento que mantiene la proporcionalidad maximizando o minimizando variables independientes en el modelo de planificación ambiental hídrica estacional dentro del intervalo de valoración concurrente de la calidad ambiental, con el objeto de definir una región de especificación y variación de la integralidad de factores ambientales.

Palabras clave: planificación ambiental, normalización, planta de tratamiento de agua residual municipal, cuenca hidrográfica
For transformation of independent variables representing a complex situation in a river basin and its interaction with the plants, wastewater treatment, the process of normalization of variables and analogous construction of a normalized vector of environmental quality is important for the abstraction of a model. Also the changes in the integrity of environmental factors in the environmental dynamics of the watershed, once defined the region. The method used in this study is documentary, explanatory and descriptive by collecting secondary information for the development of normalization of environmental quality variables. The results establish that the methods applied to maximize and minimize data set are more suited to the environmental quality variables. An analysis of normalization methods presents a procedure that keeps the proportionality maximizing or minimizing of independent variables in the model of seasonal water environmental planning within the range of concurrent assessment of environmental quality, in order to define a region specification and variation of the integrity of environmental factors.

Keywords: environmental planning, normalization, municipal treatment plant wastewater, watershed

\section{Introducción}

Un fenómeno complejo puede abstraerse y simplificarse mediante un modelo que permita representar una realidad observada. El uso de variables que aproxima los factores relevantes del fenómeno analizado puede conllevar a una representación adecuada del problema y a un análisis para la predicción de resultados. Los problemas de decisión y las variables ciertas o inciertas para la construcción de 
un modelo pueden ser simples (si hay pocas variables importantes), complejas (si hay muchas) o dinámicos (si las decisiones están interrelacionadas con el tiempo). Los factores de las variables de decisión controladas pueden ser: exógenas, es decir, fuera de alcance, e intermedias, o sea existe relación entre variables de decisión y externas; además cada modelo debe poseer medidas de desempeño (objetivos) y restricciones o limitaciones (Bonini, 2000). Un enfoque de modelo puede contener variables cualitativas dicotómicas y politómicas y variables cuantitativas discretas y continuas como variables independientes (Kaveh et al., 2015; Mori y Mahalec, 2015; Soler et al., 2012; Southworth et al., 2004; Domokos y Scheuring, 2004). Para la toma de decisiones en el área ambiental y en especial en la planificación de las cuencas hidrográficas, puede utilizarse la construcción de modelos estadísticos para datos cuantitativos. Además para datos cualitativos es posible la construcción mediante el análisis de correspondencia. En ambos casos, no es completamente necesario una estandarización o normalización de la variables que hacen parte del modelo (Peña, 2002). Sin embargo, por la consideración de tener variables independientes con diferentes unidades de medición para la representación de la calidad ambiental del fenómeno estudiado, se considera la transformación de variables mediante la normalización y un conjunto de relaciones entre las variables, y así simplificar y realizar una toma de decisión a través de la variable respuesta o dependiente. En el caso de las variables continuas, las unidades de medida de cada una de ellas son diferentes y corresponden a cada criterio ambiental. Para que sean comparables en magnitud (Barba-Romero, 1987), la normalización de las variables puede ser una alternativa válida para la construcción del modelo de planificación ambiental hídrica estacional MPAHE, dado que cada variable independiente en el modelo $r_{\mathrm{ij}}$ corresponde a cada componente ambiental $i$ a un criterio ambiental $j$ y serán interrelacionados entre sí. El vector normalizado de calidad ambiental $\left(\mathrm{CA}_{1} \ldots\right.$ $\mathrm{CA}_{\mathrm{i}}$...., $\mathrm{CA}_{\mathrm{m}}$ ) corresponde a una simplificación de vectores (Barba-Romero, 1987) para lograr una planificación adecuada de las cuencas hídricas, incluyendo la variable de las plantas de tratamiento de aguas residuales municipales PTRAM y una interrelación de la dinámica de ambiental de la cuencas hídricas.

\section{Modelo de planificación ambiental hídrica estacional MPAHE}

El ordenamiento ambiental territorial OAT como un instrumento de planificación ambiental PA es un tipo de planificación orientada a la operatividad de cuatro objetivos generales de la política ambiental: la protección de componentes o ámbitos especialmente vulnerables o estimados como valiosos por la sociedad; la reparación de componentes o ámbitos dañados; la optimización de los usos de la tierra para minimizar cargas ambientales y la prevención y descarga con relación al bienestar y la salud humana (Aronoff, 1989; Leitmann, 1999; de Roo y Miller, 2004; Jasanoff, 2004; Sujual et al., 2015). Lo anterior tiene en cuenta aspectos tales como el estudio de impacto ambiental, planificación de áreas protegidas, planes de descontaminación, aportes ambientales a la planificación urbana y ordenamiento territorial y planificaciones sectoriales para la protección o reparación de ciertos componentes o ámbitos (descontaminación, recuperación de ríos y cuerpos de aguas, manejo de biótopos, sistemas de áreas verdes, etc.), que convencionalmente incluye la dimensión ambiental DA y la protección, manejo y ordenamiento de las cuencas hidrográficas PMOCH (OEA, 1978; López et al., 2012; Villavicencio et al., 2011). El MPAHE a su vez analiza el inventario del recurso hídrico superficial y subterráneo IRH, la influencia de la población que ejerce presión en las cuencas hídricas mediante las actividades socioeconómicas de los municipios $\mathrm{M}$, las zonas de protección de bosques de los nacimientos de agua $\mathrm{ZPB}$, las acciones de protección en las cuencas hídricas APCH y la oferta y demanda del recurso hídrico ODRH. De esta forma se propone lo siguiente:

- Si el ordenamiento ambiental territorial puede ser OAT $f(\mathrm{PA})$ y PA $f(\mathrm{PMOCH}, \mathrm{DA})$, se puede considerar por tanto una representación de $\mathrm{CH}=\{\mathrm{IRH}, \mathrm{M}, \mathrm{ZPB}$, $\mathrm{APCH}, \mathrm{ODRH}\}$.

- Se puede realizar un planteamiento de un MPAHE considerando $\mathrm{N}=\{$ PTRAM, CA, EA, $\mathrm{CH}\}$ donde CA es la carga ambiental y EA es el efecto ambiental.

- Se integra la variabilidad climática estacional VCE, dado que se considera existen registros de datos entre 6 a 12 meses y se establece un mediano periodo de 
planificación (García et al., 2012; Ruiz y Franco, 2009; Zúñiga et al., 2012).

También se considera una variable cualitativa denominada perturbación Per, siendo considerada como una variable controlada que afecta adversamente el valor de salida del sistema. En la cuantificación, la medición consiste en conocer si la cuenca hídrica tiene política de gestión integral de recurso hídricos, plan de ordenamiento y manejo, control de contaminantes emergentes, plan de desarrollo municipal, control en los factores de salud por la exposición a la contaminación hídrica y si afecta el sector productivo.

Se establece que VCE es una función de la cuenca hídrica, lo cual se comprueba y valida si (VCE, CH):PAHE(CVE, $\mathrm{CH})$, se puede llegar a una planificación adecuada de las cuencas hídricas y una interrelación de las dinámicas ambientales.

Por ello, se pueden plantear alcances de análisis tales como:

- Identificar y analizar factores que influyen en el comportamiento de las plantas de tratamiento de aguas residuales municipales en términos de variables como: caudal Q, $\mathrm{DBO}_{5}, \mathrm{SST}, \mathrm{N}_{-} \mathrm{NO}_{2}, \mathrm{P}_{\text {total }}$, carga de lodos $\mathrm{CL}$, energía eléctrica consumida EEC, emisión de $\mathrm{CO}_{2} \mathrm{ECO}$.

- Análisis de la información de la calidad del agua de la cuenca hídrica; $\mathrm{DBO}_{5}, \mathrm{SST}, \mathrm{N} \mathrm{NO}_{2}, \mathrm{P}_{\text {total }}$ información de precipitación $\mathrm{P}$ y la perturbación Per.

- Según lo anterior, se integra en un MPAHE $f\{\mathrm{Q}, \mathrm{DBO}$, $\left.\mathrm{SST}, \mathrm{N} \mathrm{NO}_{2}, \mathrm{P}_{\text {total }}, \mathrm{CL}, \mathrm{EEC}, \mathrm{ECO}, \mathrm{P}, \mathrm{Per}\right\}$ para conocer sus interrelaciones con la dinámica ambiental de las cuencas hídricas.

De acuerdo a lo anterior, el objetivo del presente artículo, es realizar un análisis de los diferentes métodos de normalización de variables que puedan servir para la construcción de un Modelo de Planificación Ambiental Hídrica Estacional MPAHE ( $Q, D B O_{5}, S S T, N_{2}, P_{\text {Totap }}$ $C L, E E C, E C O, P e r)$ en una cuenca hídrica, el cual es un modelo del tipo colaborativo para la toma de decisiones que exige variables normalizadas.

\section{Metodología}

El tipo de investigación desarrollada tuvo la característica documental y explicativa (Hurtado, 1998; Balestrini, 2001). Sin embargo, según el análisis y alcance de los resultados, también es del tipo descriptivo (Vergel, 1997), dado que se detallan algunas características o propiedades de la normalización de variables de calidad ambiental. La técnica de recolección de información fue orientada según los datos obtenidos de monitoreo de la calidad del agua del río Bogotá. Los datos de calidad del agua de los ríos, fueron tomados de los reportes de frecuencia de muestreos trimestrales (periodo 2009 a 2014) y los parámetros fueron determinados con los métodos analíticos (Spellman, 2013): $D B O_{5}$, prueba de 5 días $5210 \mathrm{~B}$; SST, secado a $103-$ $105^{\circ} \mathrm{C} 2540 \mathrm{D} ; \mathrm{N} \mathrm{NO}_{2}$, colorimétrico $4500-\mathrm{NO}_{2} \mathrm{~B} ; P_{\text {Total }}$, ácido ascórbico 4500-P E; y caudal, método volumétrico (EPA, 1983). Los muestreos fueron del tipo puntual para los datos de calidad del agua en los ríos y del tipo compuesto a la salida de las PTRAM según lo establecido por el IDEAM (2002). Los parámetros: CL, $0.01 \frac{\mathrm{kg} \mathrm{SSV}}{\mathrm{kg} \mathrm{DBO}}<$ factor emisión lodos $\leq 0.8 \frac{\mathrm{kg} \mathrm{SSV}}{\mathrm{kg} \mathrm{DBO}} ; \mathrm{EEC}, 15 \mathrm{w} / \mathrm{m}^{3}<$ factor consumo energía eléctrica $\leq 88 \mathrm{~W} / \mathrm{m}^{3}$; emisión de $\mathrm{CO}_{2}$, factor de emisión de $0.6 \mathrm{~kg} \mathrm{CH}_{4} / \mathrm{kg} \mathrm{DBO} ; 0.005 \mathrm{~kg} \mathrm{~N} \mathrm{~N}_{2} \mathrm{O} /$ $\mathrm{kg} \mathrm{N}$ y factores equivalentes $\mathrm{CH}_{4}$ y $\mathrm{N}_{2} \mathrm{O}$; fueron estimados según referencias de la literatura para cada tecnología de la PTRAM. Los datos de precipitación fueron tomados de registros mensuales desde 2009 a 2014 por cada estación cercana a cada PTRAM. Además, los intervalos construidos para la normalización de variables de calidad ambiental, se fundamentaron en la normatividad establecida para descargas de vertimientos tratados a cuerpos de aguas superficiales.

\section{Métodos de normalización}

Existen varios procedimientos de normalización de variables y/o evaluaciones que establecen que existe un modelo $r_{\text {ij }}$ para cada componente ambiental $i$ y criterio ambiental $j$. En la simplificación de la notación se puede llamar $\left(V_{1} \ldots V_{\mathrm{i}} \ldots, V_{\mathrm{m}}\right)$ al vector de variables de las alternativas correspondiente a un cierto criterio genérico, el cual se puede transformar al vector normalizado de una variable $\left(v_{1}, \ldots, v_{\mathrm{i}}, \ldots v_{\mathrm{m}}\right)$ escala normalizada $0<v_{\mathrm{i}} \leq 1$, donde se supone que $V_{\mathrm{i}} \geq 0$ para todo $i$ (Barba-Romero, 1987). De forma análoga se llamará en este caso el vector normalizado de calidad ambiental CA $\left(C A_{1}, \ldots, C A_{\mathrm{i}}, \ldots\right.$ $C A_{\mathrm{m}}$ ), el cual corresponde a una simplificación de vectores 
y escala normalizada $0<C A_{\mathrm{i}} \leq 1$.

El método A de normalización consiste en mantener la proporcionalidad $\frac{V_{i}}{V_{k}}=\frac{v_{i}}{v_{k}}$ para todo $i \mathrm{y} k$, con la expresión $v_{i}=\frac{V_{i}}{\text { Máxima } V_{i}}$ usando un criterio de beneficio (maximizar) y el método $\mathrm{B}$ usando un criterio de costo (minimizar) $v_{i}=\frac{\text { Minimo }_{i}}{V_{V}}$ (García, 2009; Zeleny, 1982). El método $\mathrm{C}$ consiste en no mantener la proporcionalidad con un criterio de beneficio (maximizar) $v_{i}=\frac{V_{i}-\text { Minimo }_{i}}{{\text { Máxima } V_{i}-\text { Minimo }_{i}}_{\text {Cosing }}}$ y el método $\mathrm{D}$ con un criterio de costo (minimizar) $v_{i}=\frac{\text { Máximo } V_{i}-V_{i}}{\text { Máxima } V_{i}-\text { Minimo }_{i}} \quad$ (García, 2009; Márquez, 1999; Garduño et al., 2013). El método E permite condensar valores pequeños de $v_{\mathrm{i}}$ con la expresión $v_{i}=\frac{V_{i}}{\sum_{i=1}^{k} V_{i}}$ (García, 2009; Boyacioglu, 2012). El método $\mathrm{F}$ permite las comparaciones adimensionales de los vectores criterio del problema con la expresión $v_{i}=\frac{V_{i}}{\left(\sum_{i=1}^{k} V_{i}^{2}\right)^{0.5}}$ (Barba-Romero, 1987; García, 2009; Pomerol y Barba-Romero, 2012).

Sin embargo, se pueden construir coeficientes que proporcionan índices ambientales IA relativos que son magnitudes que miden una proporcionalidad y pueden ser representados en términos de la calidad ambiental en unidades estandarizadas como se mencionó anteriormente. Se puede expresar conceptualmente por ejemplo $I A=\frac{\text { calidad previa }}{\text { estándar de calidad ambiental }} ; I A=\frac{\text { cantidad de emisión }}{\text { estándar de emisión }}$ $I A=\frac{\text { calidad previa }}{\text { media temporal o espacial }}$ (Gross y Hajek, 1998; Gómez y Gómez, 2011).

Por otro lado, se puede construir un vector normalizado de calidad ambiental utilizando el método $\mathrm{G}$ de regresión de mínimos cuadrados (lineal: $v_{i}=a+b V_{i}$, potencia: $v_{i}=$ $a V_{i}^{b}$ o exponencial: $v_{i}=a e^{b v_{i}}$ ) y utilizando el coeficiente de correlación (Pearson) para determinar la relación entre variables; un criterio significativo es tener $0.9<r \leq 1$, lo cual se considera como una excelente correlación de datos (Johnson, 2000; Hair, 1999; Agresti y Kateri, 2011; Walpole et al., 1999). Con los datos de cada variable independiente se utilizaron los métodos A, B, C, D, E, F y $\mathrm{G}$, los cuales fueron aplicados con los datos secundarios obtenidos de las cuencas hídricas y plantas de tratamiento de aguas residuales municipales PTRAM.

\section{Análisis de resultados}

La Tabla 1 muestra rangos de la calidad del agua en cuerpos superficiales en países tropicales (Cude, 2001; Grafe, 2002; Sarkar y Abbasi, 2006; Yisa y Jimoh, 2010; Liou et al., 2004).

Tabla 1: Rango de trabajo de las variables.

\begin{tabular}{|c|c|c|}
\hline $\begin{array}{c}\text { Varia- } \\
\text { ble }\end{array}$ & \begin{tabular}{|l|} 
Interva- \\
lo, mg/L
\end{tabular} & Calidad ambiental, mg/L \\
\hline DBO & 1 y 8 & $\begin{array}{l}\mathrm{Si} \mathrm{DBO}_{5} \leq 8, \text { entonces } C A=100 e^{\mathrm{DBO}-0.1993} \\
\text { si } \mathrm{DBO}_{5} \geq 8, \text { entonces } C A=0.1\end{array}$ \\
\hline SST & 1 y 220 & $\begin{array}{l}\text { Si SST } \leq 40 \text {, entonces } C A=1.0 ; \text { si } 40 \leq \\
\mathrm{SST} \leq 220 \text {, entonces, } C A=142.6 e^{\mathrm{SST}-1.1075} \\
\text { si SST } \geq 200 \text { entonces } \mathrm{CA}=0.1\end{array}$ \\
\hline $\begin{array}{l}\mathrm{N}- \\
\mathrm{NO}_{3}\end{array}$ & 0.25 y 3 & $\begin{array}{l}\mathrm{Si} \mathrm{N}-\mathrm{No}_{3} \leq 3, \text { entonces } C A=100 e^{\mathrm{N}-0.4605} \\
\text { si N- } \mathrm{NO}_{3} \geq 3 \text {, entonces } \mathrm{CA}=0.1\end{array}$ \\
\hline$P_{\text {TOtAL }}$ & $\begin{array}{c}0.025 \\
\text { y } 3\end{array}$ & $\begin{array}{l}\text { Si } \mathrm{P}_{\text {TOTAL }} \leq 3, \text { entonces } C A=100-299.5 P \\
0.1384 P^{2} ; \text { si } P_{\text {TOTAL }} \geq 0.25 \text {, entonces } \\
\mathrm{CA}=0.1\end{array}$ \\
\hline
\end{tabular}

Sin embargo, los rangos de concentraciones corresponden a la integración entre el criterio de descarga normativo de residuos líquidos municipales a cuerpos de agua y los resultados reportados de calidad del agua. Los resultados a utilizar con los métodos de normalización descritos anteriormente se describen a continuación.

En las Figuras 1, 2, 3 y 4 se aprecia la aplicación de los métodos $\mathrm{A}$ a $\mathrm{G}$, donde se relaciona la variable independiente $\mathrm{DBO}_{5}, \mathrm{~N}-\mathrm{NO}_{2}, \mathrm{SST}, \mathrm{P}_{\text {TOTAL }}$ y la dependiente $\mathrm{CA}$. El comportamiento entre las variables es similar con tendencia lineal de pendiente negativa para los métodos D y G, lo cual indica que la variable CA se adapta a las escalas determinadas de las variables independientes consideradas en el análisis y la representación de la normalización de la variable CA es válida en el intervalo de estudio de la variable independiente. Al aplicar el método $\mathrm{D}$, se considera que la variable independiente sea $V_{i} \geq 0 \mathrm{y}$ perfecciona la escala normalizada en el intervalo $0<C A_{\mathrm{i}} \leq$ 1 según el rango de datos de la variable independiente y sin embargo no conserva la proporcionalidad, lo cual puede ser solucionado al invertir el proceso de decisión (García, 2009; Pomerol y Barba-Romero, 2012). Para el método G, se observa un comportamiento lineal negativo $\left(\mathrm{DBO}_{5}, \mathrm{~N}-\right.$ $\mathrm{NO}_{2}, \mathrm{SST}, \mathrm{P}_{\text {TOTAL }}$ ) con coeficientes de correlación o Pearson $r^{2}=0.9982,1,1$ y 0.9959 , respectivamente, de relación directa. Ello indica que los valores observados en 
las variables independientes tienen una correlación denominada excelente (Agresti y Kateri, 2011; Hair, 1999) con relación a los valores de CA, es decir que la variable CA es explicada por la variación de la variables independientes, lo cual establece que hay una correlación adecuada con una tendencia clara y obedecen a un patrón lineal en el rango de análisis. Los métodos $\mathrm{A}$ y $\mathrm{C}$ presentan una escala de CA invertida que proporciona un análisis de valoración de CA en una dimensión diferente a la concepción de las escalas de las variables independientes. No es así para los métodos $\mathrm{B}, \mathrm{E}$ y $\mathrm{F}$, que presentan una agrupación de valores de CA sin la representación completa de la normalización misma de CA.

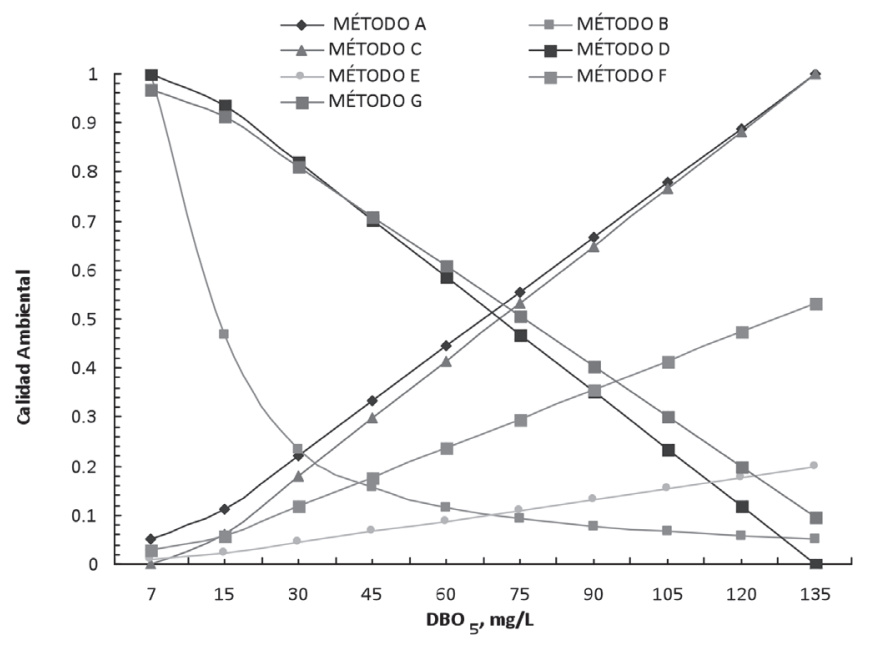

Figura 1: Calidad ambiental normalizada para el parámetro $\mathrm{DBO}_{5}$

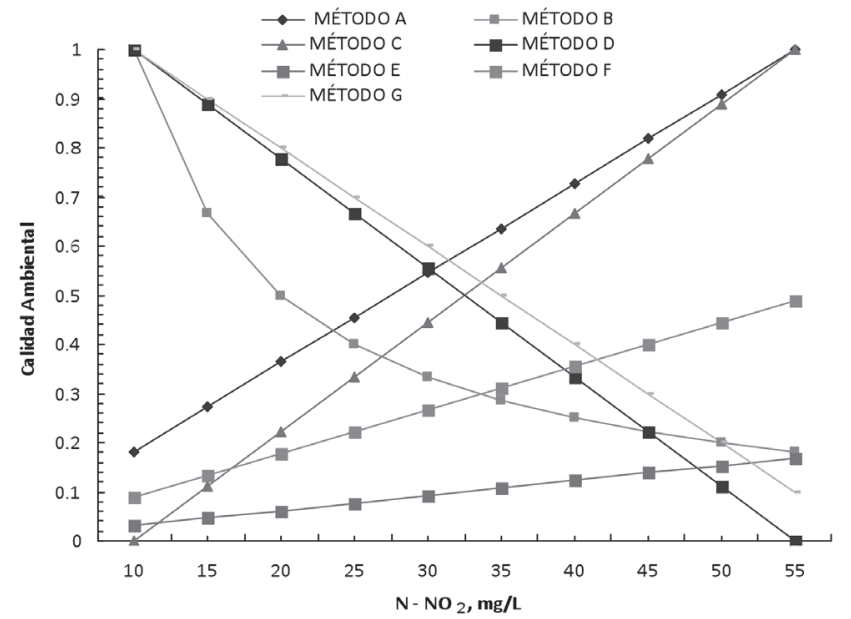

Figura 2: Calidad ambiental normalizada para el parámetro $\mathrm{N}$ $-\mathrm{NO}_{2}$

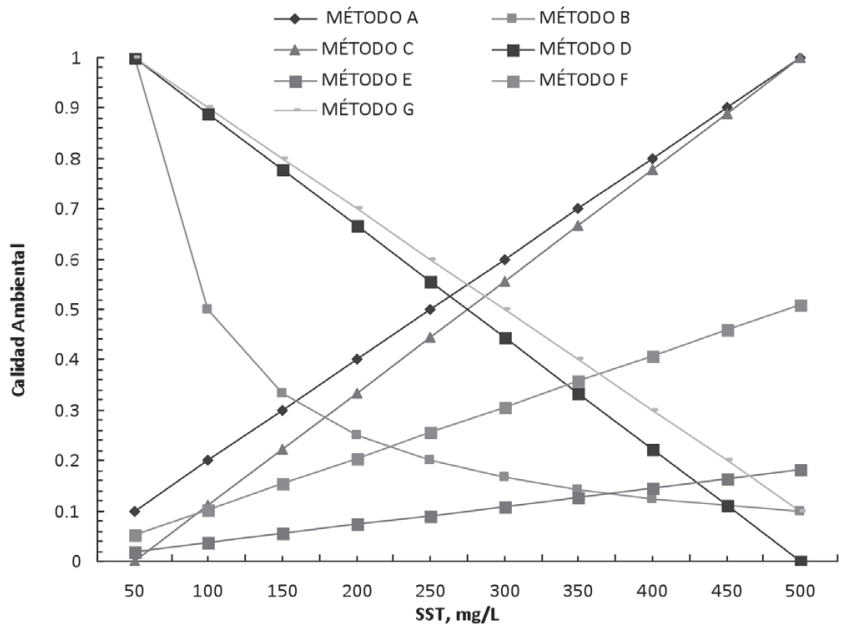

Figura 3: Calidad ambiental normalizada para el parámetro SST

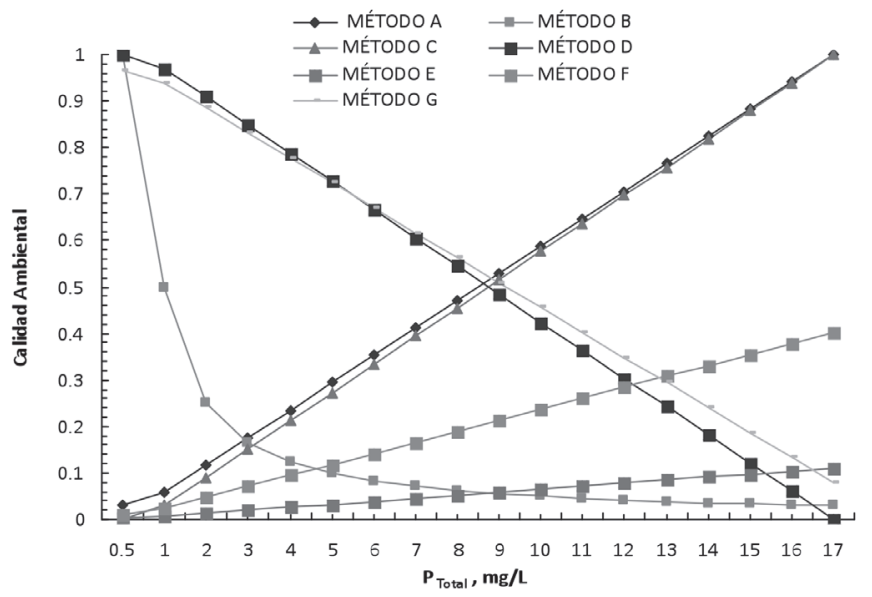

Figura 4: Calidad ambiental normalizada para el parámetro $\mathrm{P}_{\text {Total }}$

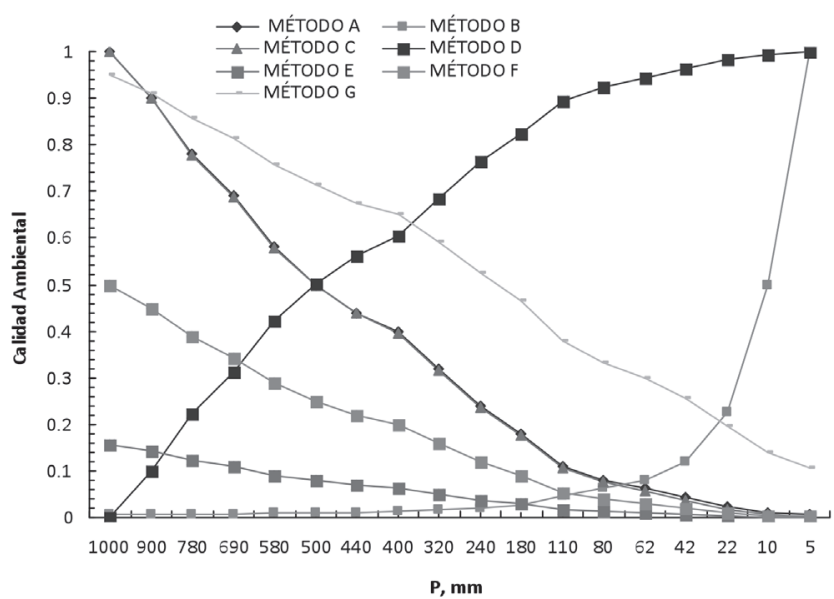

Figura 5: Calidad ambiental normalizada para el parámetro precipitación $\mathrm{P}$ 


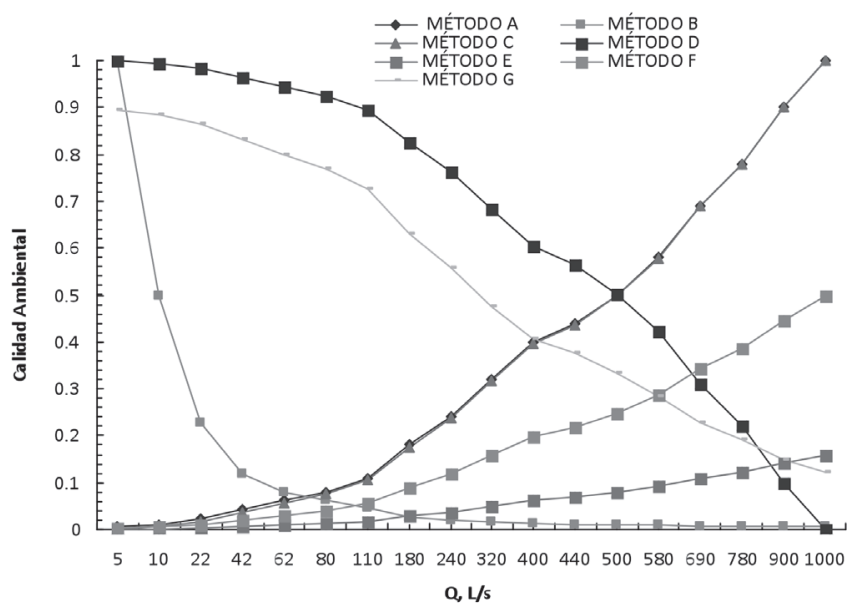

Figura 6: Calidad ambiental normalizada para el parámetro caudal PTRAM Q

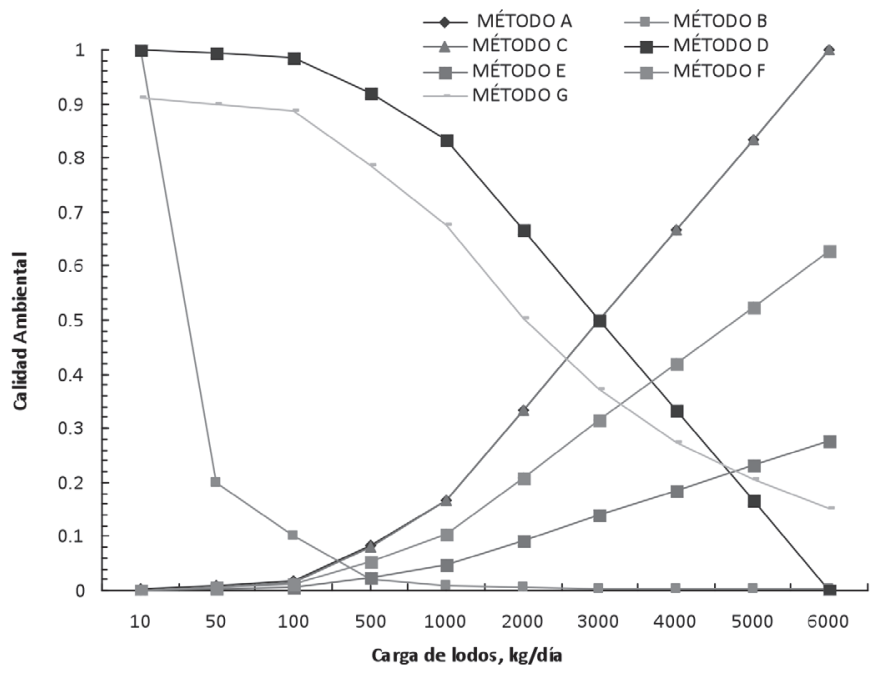

Figura 7: Calidad ambiental normalizada para el parámetro carga de lodos CL

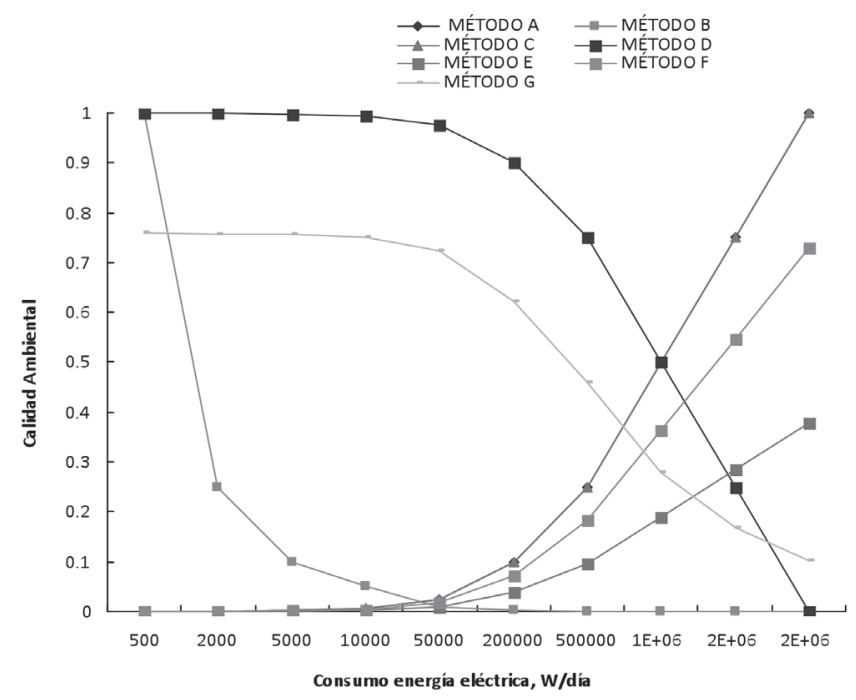

Figura 8: Calidad ambiental normalizada para el parámetro energía eléctrica consumida EEC

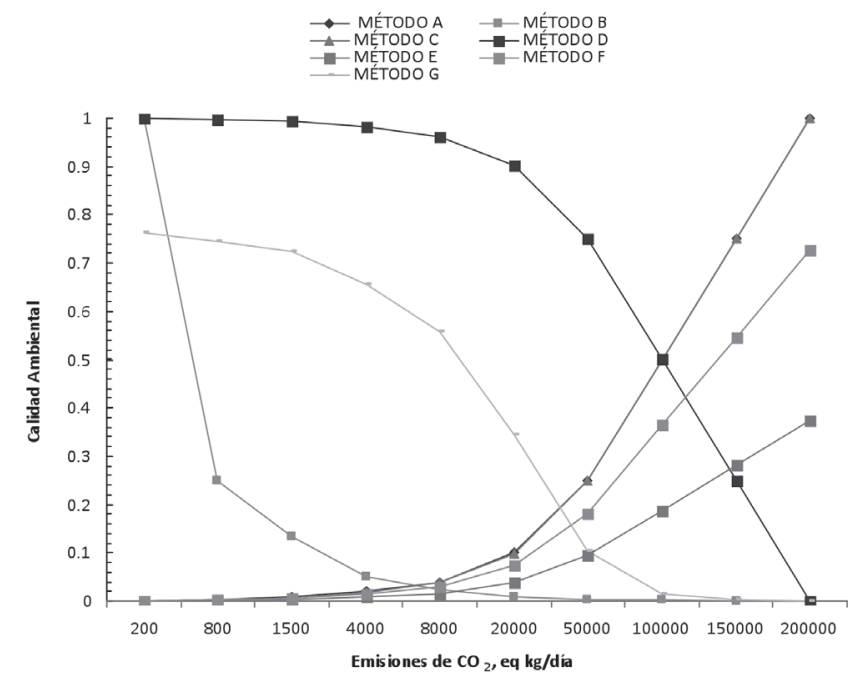

Figura 9: Calidad ambiental normalizada para el parámetro emisiones de $\mathrm{CO}_{2} \mathrm{ECO}$

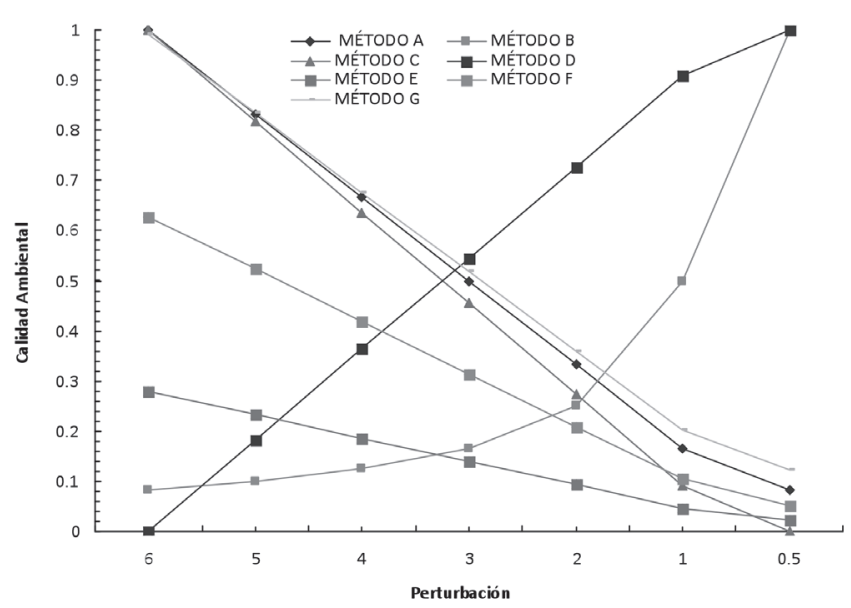

Figura 10: Calidad ambiental normalizada para el parámetro perturbación Per

En las Figuras 6, 7, 8 y 9, se aprecia que la variable dependiente CA al aplicarse los métodos $\mathrm{D}$ y $\mathrm{G}$ con las variables independientes Q, CL, EEC, ECO, presenta un adecuado comportamiento. Los métodos D y G presentan una diferencia DCA de 0.24 (ECO, EEC), 0.09 (CL) y $0.11(\mathrm{Q})$, en el valor inicial de la estimación de CA, lo cual genera una distorsión considerable en la predicción de CA según los valores concebidos en las variables independientes. Al aplicar el método $\mathrm{C}$, se considera que la variable independiente sea $V_{i} \geq 0$ y perfecciona la escala normalizada en el intervalo $0<C A_{\mathrm{i}} \leq 1$ según el rango de los datos de la variable independiente y sin embargo no conserva la proporcionalidad, el cual puede ser solucionado al invertir el proceso de decisión (García, 2009; Pomerol y Barba-Romero, 2012). Para el método 
$\mathrm{G}$, se observa un comportamiento exponencial (Q, CL, EEC, ECO) y con coeficientes de correlación o Pearson $r^{2}=0.9871,0.9632,0.9499$ y 0.9512 , respectivamente, lo cual indica que los valores observados en las variables independientes tienen una correlación denominada excelente (Agresti y Kateri, 2011; Hair, 1999) con relación a los valores de la CA.

En las Figuras 5 y 10, CA en los métodos $\mathrm{C}$ y $\mathrm{G}$ es muy similar y por ello existe una correlación entre sí muy significativa y una diferencia mínima entre métodos. Esto considera que la respuesta del vector de normalización está directamente relacionado con la variable independiente e influye en CA y la tendencia es una función cóncava. Al aplicar el método $\mathrm{C}$, se considera que la variable independiente $V_{i} \geq 0$ perfecciona la escala normalizada en el intervalo $0<C A_{\mathrm{i}} \leq 1$ según el rango o intervalo de los datos de la variable independiente y sin embargo no conserva la proporcionalidad, lo cual puede ser solucionado al invertir el proceso de decisión (García, 2009; Pomerol y Barba-Romero, 2012). Para el método G, se observa un comportamiento exponencial (P) y lineal negativa (Per) con coeficientes de correlación o Pearson $r^{2}=0,9948$ y 0,9933 , respectivamente, de relación directa. Esto indica que los valores observados en las variables independientes tienen una correlación denominada excelente (Agresti y Kateri, 2011; Hair, 1999) con relación a los valores de la CA. Al aplicar los métodos B y D, se observa un comportamiento inverso en la función predictiva de CA. En los métodos A, E y F, la estimación de la CA no alcanza los valores de representación completa de la normalización misma de la variable dependiente.

Las variables normalizadas o transformadas, pueden ser del tipo lineal $\left(C A=a+b V_{i}\right)$ y no lineal $\left(C A=a V_{i}^{b} ; C A=a e^{b v_{i}}\right)$, función lineal y no lineal segmentada (Abbassi y Abbasi, 2012). Por ello al utilizar el método de mínimos cuadrados, la regresión fue lineal para las variables independientes $\mathrm{DBO}_{5}, \mathrm{SST}, \mathrm{N}-\mathrm{NO}_{2}, \mathrm{P}_{\text {Total }}$, Per, mientras que para las variables independientes la regresión fue exponencial para $\mathrm{Q}, \mathrm{CL}, \mathrm{EEC}, \mathrm{ECO}$ y regresión de potencia para $\mathrm{P}$.

Una valoración concurrente de la normalización de CA considera que en el intervalo $0.8<C A_{\mathrm{i}} \leq 1$, el estado o calidad del recurso hídrico es muy bueno; en el rango de $0.6<C A_{\mathrm{i}} \leq 0.8$, es bueno; en el rango de $0.4<C A_{\mathrm{i}} \leq$ 0.6 , es regular; en el rango de $0.2<C A_{\mathrm{i}} \leq 0.4$, es malo; y en el rango de $0<C A_{\mathrm{i}} \leq 0.2$, es pésimo o muy malo. Lo anterior servirá para la toma de decisiones en la intervención en el cuerpo de agua con medidas simples o complejas, seguimiento y control, e inversiones para el mejoramiento o mantenimiento del recurso hídrico. Esto conlleva a establecer que el vector de normalización de CA para $\mathrm{DBO}_{5}, \mathrm{SST}, \mathrm{N}-\mathrm{NO}_{2}, \mathrm{P}_{\text {Total }}$ y Per, los métodos A, B, C, D y G, sólo con regresión lineal negativa, se considera que a mayor concentración mejor es la CA dentro del rango de análisis. En el caso de Per se considera que es mejor cuando existen los instrumentos de gestión para la cuenca hídrica. Sin embargo para los métodos E y F, la normalización no alcanza los resultados indicados en la valoración concurrente de CA. Para las variables Q, P, CL, EEC y ECO, los métodos A, B, C y D se consideran mejor para el análisis del fenómeno. $\mathrm{P}$ es diferente a la consideración, dado que a mayor precipitación, se presenta dilución natural del contaminante, es decir, que a menor valor de la variable independiente $\mathrm{CA}$ es mejor, no así, para los métodos E, F y G, dado que no alcanzan la valoración completa en la escala de CA. Por ello se excluyen los métodos E, F y G (sólo con regresión exponencial y de potencia), por no alcanzar a representar la valoración concurrente de CA en su intervalo de 0 a 1. En la Tabla 2 se presentan los criterios para la utilización del vector normalizado para la CA por cada parámetro representativo del fenómeno a analizar dentro del MPAHE, del cual se propone la utilización de un método normalizado de minimización o maximizando la variable independiente (Gharibi et al., 2012) o la aplicación de un método de regresión lineal o exponencial o de potencia.

\section{Conclusiones}

En la normalización de variables vectoriales continuas para el Modelo de Planificación Ambiental Hídrica Estacional MPAHE, los métodos $\mathrm{C}$ y D de proporción de la variable dentro del rango de operación o trabajo, son los que más se ajustan a la representación del intervalo o rango de la variable independiente analizada. Al utilizar el método G (ajuste de mínimos cuadrados), se observó que cuando la regresión es lineal negativa, el ajuste y concordancia es alta, con los métodos C y D. Sin embargo, cuando la regresión es exponencial y de potencia, se presentan restricciones en su aplicabilidad, dado que no alcanza el intervalo de la valoración concurrente de calidad 
Tabla 2: Resumen de métodos normalizados aplicados a la calidad ambiental en un MPAHE

\begin{tabular}{|c|c|c|c|c|}
\hline Unidad & Intervalo & \multicolumn{2}{|c|}{ Método normalizado } & Condiciones \\
\hline $\mathrm{mg} / \mathrm{L}$ & $7 \leq \mathrm{DBO}_{5} \leq 135$ & $C A_{i}=\frac{\max D B O_{i}-D B O_{i}}{\max D B O_{i}-\min D B O_{i}}$ & $\begin{array}{c}C A_{i}=1.0157-0.0068 D B O \\
r^{2}=0.9982\end{array}$ & $\begin{array}{c}\text { Si } D B O_{5} \geq 135, \\
C A_{\mathrm{i}}=0.1\end{array}$ \\
\hline $\mathrm{mg} / \mathrm{L}$ & $10 \leq N_{\mathrm{NO} 2} \leq 55$ & $C A_{i}=\frac{\max N_{i}-N_{i}}{\max N_{i}-\min N_{i}}$ & $\begin{array}{c}C A_{i}=1.2-0.02 N \\
r^{2}=1\end{array}$ & $\begin{array}{l}\text { Si } N_{\mathrm{NO} 2} \geq 55 \\
\quad C A_{\mathrm{i}}=0.1\end{array}$ \\
\hline $\mathrm{mg} / \mathrm{L}$ & $50 \leq S S T \leq 500$ & $C A_{i}=\frac{\max S S T-S S T}{\max S S T-\min S S T}$ & $\begin{array}{c}C A_{i}=1.1-0.002 S S T \\
r^{2}=1\end{array}$ & $\begin{array}{c}\text { Si } S S T \geq 500, \\
\quad C A_{\mathrm{i}}=0.1\end{array}$ \\
\hline $\mathrm{mg} / \mathrm{L}$ & $0.5 \leq P_{\mathrm{T}} \leq 17$ & $C A_{i}=\frac{\max P_{T i}-P_{T i}}{\max P_{T i}-\min P_{T i}}$ & $\begin{array}{c}C A_{i}=0.9927-0.0537 P_{\text {total }} \\
r^{2}=0.9959\end{array}$ & $\begin{array}{l}\text { Si } P_{\mathrm{T}} \geq 17 \\
C A_{\mathrm{i}}=0.1\end{array}$ \\
\hline $\mathrm{mm}$ & $5 \leq P \leq 100$ & $C A_{i}=\frac{\max P-P}{\max P-\min P}$ & & $\begin{array}{l}\text { Si } P \leq 5 \\
C A_{\mathrm{i}}=0.1\end{array}$ \\
\hline $\mathrm{L} / \mathrm{s}$ & $5 \leq Q \leq 1000$ & $C A_{i}=\frac{\max Q_{i}-Q_{i}}{\max Q_{i}-\min Q_{i}}$ & & $\begin{array}{l}\text { Si } Q \geq 1000 \text {, } \\
\quad C A_{\mathrm{i}}=0.1\end{array}$ \\
\hline $\mathrm{kg} / \mathrm{día}$ & $10 \leq C L \leq 6000$ & $C A_{i}=\frac{\max C L_{i}-C L_{i}}{\max C L_{i}-\min C L_{i}}$ & & $\begin{aligned} \text { Si } C L & \geq 6000 \\
C A_{\mathrm{i}} & =0.1\end{aligned}$ \\
\hline $\mathrm{kW} /$ día & $0.5 \leq E E C \leq 2000$ & $C A_{i}=\frac{\max E E C_{i}-E E C_{i}}{\max E E C_{i}-\min E E C_{i}}$ & & $\begin{array}{c}\text { Si } E E C \geq 2000, \\
C A_{\mathrm{i}}=0.1\end{array}$ \\
\hline eq ton día & $0.2 \leq E C O \leq 200$ & $C A_{i}=\frac{\max E C O_{i}-E C O_{i}}{\max E C O_{i}-\min E C O_{i}}$ & & $\begin{array}{c}\text { Si } E C O \geq 200 \\
C A_{\mathrm{i}}=0.1\end{array}$ \\
\hline- & $0.5 \leq \operatorname{Per} \leq 6$ & $C A_{i}=\frac{P e r_{i}-\min P e r_{i}}{\max P e r_{i}-\min P e r_{i}}$ & $\begin{array}{c}C A_{i}=0.0431+0.1581 \mathrm{Per} \\
r^{2}=0.9933\end{array}$ & $\begin{array}{l}\text { Si } P e r \leq 0.5 \text {, } \\
\quad C A_{\mathrm{i}}=0.1\end{array}$ \\
\hline
\end{tabular}

ambiental. Un beneficio de normalizar vectores o variables como la calidad ambiental, es que se define una región de especificación y variación de la integralidad de factores ambientales en la dinámica ambiental de cuencas hídricas. Con ello, es posible ponderar de manera concurrente o en paralelo, una valoración cuantificable y cualificable de la planificación ambiental en las cuencas hídricas. Se concibe el MPAHE incorporando variables o vectores normalizados en función de la calidad ambiental, lo cual permite flexibilizar y encausar una toma de decisión de ordenamiento y planificación adecuada de unas cuencas hídricas.

\section{Agradecimientos}

El primer autor agradece a la Escuela de Ingeniería Civil Industrial y al Departamento de Ingeniería Civil y Ambiental de la Universidad del Bío-Bío por el apoyo académico brindado en el desarrollo de la pasantía de investigación. De igual forma, se agradece a la Agencia de Cooperación Internacional AGCI de Chile por la asignación de la beca del programa de movilidad estudiantil y académica de la Alianza del Pacífico, para la realización de pasantía de investigación en la Universidad del Bío-Bío.

\section{Referencias}

Abbassi, T. and Abbasi, S.A. (2012). Water quality indices. Elsevier, Oxford, UK

Agresti, A. and Kateri, M. (2011). Categorical data analysis. Springer

Aronoff, S. (1989). Geographic information systems: a management perspective. WDL Publications, Ottawa, Canada

Balestrini, M. (2001). Cómo se elabora el proyecto de investigación. OBL, Caracas 
Barba-Romero, S. (1987). Panorámica actual de la decisión multicriterio discreta. Investigaciones Economicas 11(2), 279 - 308

Bonini, C. (2000). Análisis cuantitativo para los negocios. Mc Graw Hill Interamericana, Bogotá

Boyacioglu, H. (2012). Utilization of environmetric \& index methods as water quality comparative assessment tools focusing on heavy metal content. Archives of Environemental Protection 38, No 3, 17 - 28

Cude, C.G. (2001). Oregon water quality index: a tool for evaluating water quality management effectivenss. Journal of the American Water Resources Association JAWRA 37(1), 125 - 137

de Roo, G. and Miller, D. (2004). Integrating city planning and environmental improvement: practicable strategies for sustainable urban development. Gower publishing

Domokos, G. and Scheuring, I. (2004). Discrete and continuous state population models in a noisy world. Journal of Theoretical Biology 227(4), 535-545

EPA (1983). Methods for chemical analysis of water and wastes. Environmental Protection Agency, USA

García, M.S. (2009). Métodos para la comparación de alternativas mediante un Sistema de Ayuda a la Decisión SAD y Soft Computing. Tesis de doctorado, Universidad Politécnica de Cartagena

García, M.C., Botero, A.P., Quiroga, F.A.B. y Robles, E.A. (2012). Variabilidad climática, cambio climático y el recurso hídrico en Colombia. Revista de Ingeniería 36, 60-64

Garduño, R., Ibarra, J.E. y Dávila, R. (2013). La medición de la competitividad en México: ventajas y desventajas de los indicadores. CIDE, México

Gharibi, H., Sowlat, M.H., Mahvi, A.H., Mahmoudzadeh, H., Arabalibeik, H., Keshavarz, M., Karimzadeh, N. and Hassani, G. (2012). Development of a dairy cattle drinking water quality index (DCWQI) based on fuzzy inference systems. Ecological Indicators 20, 228-237.

Gómez, D. y Gómez, M. (2011). Evaluación ambiental estratégica (EAE); un instrumento preventivo de gestión ambiental. Revista de Salud Ambiental 11(1-2), 9-16

Grafe, C.S. (2002). Idaho river ecological assessment framework: an integrated approach. Idaho Department of Environmental Quality, Boise
Gross, P. y Hajek, E. (1998). Indicadores de calidad y gestión ambientales. Pontificia Universidad Católica de Chile, Santiago

Hair, J.F. (1999). Análisis multivariante. Prentice Hall, Madrid

Hurtado, J. (1998). Metodología de la investigación holística. Fundacite-Sypal, Caracas

IDEAM (2002). Guía para el monitoreo de vertimientos, aguas superficiales y subterráneas. Bogotá: Instituto de Hidrología, Meteorología y Estudios Ambientales. Ministerio de Ambiente, Vivienda y Desarrollo Territorial - República de Colombia Jasanoff, S. (2004). Earthly politics: local and global in environmental governance. MIT press

Johnson, D. (2000). Métodos multivariados aplicados al análisis de datos. Thomson, México

Kaveh, A., Mirzaei, B. and Jafarvand, A. (2015). An improved magnetic charged system search for optimization of truss structures with continuous and discrete variables. Applied Soft Computing 28, 400-410

Leitmann, J.L. (1999). Sustaining cities: environmental planning and management in urban design. McGraw-Hill

Liou, S.M., Lo, S.L. and Wang, S.H. (2004). A generalized water quality index for Taiwan. Environmental Monitoring and Assessment 96(1-3), 35-52

López, J.J., González, M., Scaini, A., Goñi, M., Valdenebro, J.V. y Gimena, F.N. (2012). Caracterización del modelo HEC-HMS en la cuenca de río Arga en Pamplona y su aplicación a cinco avenidas significativas. Obras y Proyectos 12, 15-30

Márquez, H. (1999). Métodos matemáticos de evaluación de factores de riesgo para el patrimonio arqueológico: una aplicación GIS del método de jeraquías análiticas de TL SAATY. Revista de Prehistoria y Arqueología de la Universidad de Sevilla 8, 21 - 37

Mori, J. and Mahalec, V. (2015). Planning and scheduling of steel plates production. Part I: Estimation of production times via hybrid Bayesian networks for large domain of discrete variables. Computers \& Chemical Engineering 79, 113-134

OEA (1978). Calidad ambiental y desarrollo de cuencas hidrográficas: un modelo para planificación y análisis integrados. Programa de las Naciones Unidas para el Medio Ambiente, Organización de los Estados Americanos, Washington D.C.

Peña, D. (2002). Análisis de datos multivariantes. Mc Graw Hill, España 